Mathematical Research Letters 8, 141-156 (2001)

\title{
LOCALIZATION FOR INVARIANT SUBMANIFOLDS
}

\author{
Mikhail Kogan
}

Abstract. Given a manifold $M$ with an equivariantly formal circle action and any invariant submanifold $W$ which does not contain fixed points of the action, we present a new localization theorem, which expresses an integration over $W / S^{1}$ in terms of an integration over the fixed points of the action. In the setting of symplectic geometry our results imply the Jeffrey-Kirwan localization theorem for the circle action.

\section{Introduction}

Let $G$ be a compact Lie group, let $M$ be an oriented compact $G$-manifold and let

$$
(p t)_{*}: H_{G}^{*}(M) \rightarrow H_{G}^{*}(p t)
$$

be the pushforward operator in equivariant cohomology. For $G$ abelian this map factors through a map

$$
i^{*}: H_{G}^{*}(M) \rightarrow H_{G}^{*}(F),
$$

with $i$ being the inclusion mapping of the fixed point set $F$ into $M$; and the explicit formula for $p t_{*}$ given by the celebrated Atiyah-Bott Berline-Vergne localization formula (see [2] and [5]). If $M$ is symplectic and the action of $G$ is Hamiltonian, Witten obtained in [18] a non-abelian variant of the localization theorem. Then in [10] Jeffrey and Kirwan showed that Witten's theorem could be interpreted as an abelian localization theorem (for Hamiltonian $G$-actions) which had a lot in common with the ABBV theorem. In particular, the ABBV theorem was the main tool in the proof of the non-abelian localization theorem.

Our purpose in this paper is to show that there is another connection between the two localization theorems. Namely, we will show that the ABBV theorem bears the same relation to the Jeffrey-Kirwan theorem that the theory of characteristic classes bears to the theory of secondary characteristic classes (i.e. Chern-Simons theory).

Our result will also clarify the role of "symplectic" in the Witten-JeffreyKirwan theorem. Namely, if $G$ acts on $M$ in a Hamiltonian fashion, $M$ is equivariantly formal by a well-known theorem of Kirwan [13], and hence any equivariant class which is in the kernel of the restriction map, $i^{*}: H_{G}^{*}(M) \rightarrow H_{G}^{*}(F)$ has to vanish. In the language of equivariant forms: if $\tau \in \Omega_{G}^{*}(M)$ is $d_{G^{-}}$closed and $i^{*} \tau$ is zero, then $\tau=d_{G} \mu$. Moreover, $\mu$ is closed on the complement of the support of $\tau$. In particular, if $\tau$ is the Thom form of a $G$-invariant submanifold,

Received September 14, 1999. 
$W$, of $M$ with no fixed points, $\tau$ is closed on $M-W$ and hence defines a cohomology class $[\mu] \in H_{G}^{*}(M-W)$. (This is the "secondary characteristic class" phenomenon that we referred to above. We will show that it is exactly this phenomenon which enables one to get a localization theorem of Jeffrey-Kirwan type.) We'll now make these remarks more precise:

We are given an action of a compact abelian group $G$ on an oriented compact connected manifold $M$. We assume that $M$ is equivariantly formal (see Definition 2.4) which, in particular, means that the equivariant cohomology of $M$ is injected into the equivariant cohomology of the fixed point set. Suppose we also have a subgroup $S^{1} \subset G$ and an invariant compact connected oriented submanifold $I: W \rightarrow M$, such that $S^{1}$ acts freely on $W$. We denote by $X=W / S^{1}$. We always have the isomorphism $c: H_{G}^{*}(W) \rightarrow H_{G / S^{1}}^{*}(X)$, and we can construct the $\operatorname{map} \kappa: H_{G}^{*}(M) \stackrel{I^{*}}{\rightarrow} H_{G}^{*}(W) \stackrel{c}{\rightarrow} H_{G / S^{1}}^{*}(X)$. This map is an analogue of the Kirwan map in symplectic geometry.

For an equivariantly closed form $\alpha \in \Omega_{G / S^{1}}^{*}(X)$ and an invariant tubular neighborhood $U$ of $W$, let $\beta$ be the equivariantly closed form which represents the pushforward cohomology class $I_{*} c^{-1}([\alpha])$ on $U$. $\beta$ is compactly supported inside $U$ and could be thought of as a form on $M$. Since we can assume that the support of $\beta$ does not intersect the fixed point set of the $S^{1}$ action, we can choose $\mu \in \Omega_{G}^{*}(M)$ such that $d_{G} \mu=\beta$. Let $i_{j}: F_{j} \rightarrow M$ be the connected components of the fixed point set of the $S^{1}$ action (notice that we are working with fixed points of the $S^{1}$ action rather than the $G$ action).

Theorem 3.2. For any equivariantly closed forms $\alpha \in \Omega_{G / S^{1}}^{*}(X)$ and $\omega \in$ $\Omega_{G}^{*}(M)$ such that $\operatorname{deg} \alpha+\operatorname{deg} \omega \leq \operatorname{dim} W$

$$
(-1)^{\operatorname{deg} \omega+1} \int_{X} \frac{\kappa([\omega])[\alpha]}{e(W)}=\sum_{F_{j}} \int_{F_{j}} \frac{\left[i_{j}^{*}(\omega \mu)\right]}{e\left(N F_{j}\right)},
$$

where $e(W)$ is the equivariant Euler class of the circle bundle $W \rightarrow X$ and $e\left(N F_{j}\right)$ are the equivariant Euler classes of the normal bundles $N F_{j}$ of $F_{j}$.

Remark 1.1. In the above theorem, $[\beta]$ should be thought of as a characteristic class, which turns out to be trivial. In analogy with Chern-Simons secondary invariants, this allows us to think of the restrictions of $\mu$ to the fixed points $F_{j}$ as secondary characteristic classes.

We will define residues in Definition 4.2 and using them prove the following theorem which in the setting of symplectic geometry is a special case of the Jeffrey-Kirwan localization theorem for the circle action.

Theorem 4.3. For any equivariantly closed forms $\alpha \in \Omega_{G / S^{1}}^{*}(X)$ and $\omega \in$ $\Omega_{G}^{*}(M)$

$$
(-1)^{\operatorname{deg} \omega} \int_{X} \kappa([\omega])[\alpha]=\sum_{F_{j}} \int_{F_{j}} \operatorname{Res} \frac{\left[i_{j}^{*}(\omega \mu)\right]}{e\left(N F_{j}\right)}
$$

where $e\left(N F_{j}\right)$ are the equivariant Euler classes of the normal bundles $N F_{j}$ 's. 
The organization of the paper is as follows. In Chapter 2 we review basic facts about equivariant cohomology of torus actions. In Chapter 3 we prove Theorem 3.2. Chapter 4 provides a residue version of our result. We explain how the standard localization theorem for the circle action follows from Theorem 4.3, give some non symplectic examples and show how these results can be generalized to group actions on orbifolds.

\section{Preliminaries in equivariant cohomology}

Let a compact abelian group $G$ act on a manifold $M$. The Cartan model for equivariant cohomology is the complex $\Omega_{G}^{*}(M)=S^{*}\left(\mathfrak{g}^{*}\right) \otimes \Omega^{*}(M)^{G}$, where: $\Omega^{*}(M)^{G}$ is the algebra of all invariant de Rham forms on $M ; \mathfrak{g}^{*}$ is the dual of the Lie algebra of $G$; and $S^{*}\left(\mathfrak{g}^{*}\right)$ is the ring of complex valued polynomials on $\mathfrak{g}$. Given a basis $x_{a}$ of $\mathfrak{g}$, we get the dual basis $u_{a}$ of $\mathfrak{g}^{*}$, which we will think of as a set of generators for $S^{*}\left(\mathfrak{g}^{*}\right)$. (The degree of each $u_{a}$ is equal to 2). For each $x_{a}$ we can construct the vector field $X_{a}(p)=\left.\frac{\partial}{\partial t}\right|_{t=0} \exp \left(t x_{a}\right)(p)$ where $p$ is a point on $M$. We denote by $i_{a}$ the contraction with respect to $X_{a}$. The equivariant differential is defined as $d_{G}=1 \otimes d+\sum u_{a} \otimes i_{a} . \Omega_{G}^{*}(M)$ together with $d_{G}$ define the equivariant cohomology $H_{G}^{*}(M)$. It is clear that equivariant cohomology of a point is $S^{*}\left(\mathfrak{g}^{*}\right)$. For an equivariantly closed form $\alpha$, we define $[\alpha]$ to be the cohomology class it represents.

Given an invariant map $f: M \rightarrow N$ between two manifolds with $G$ actions it is easy to define the pullback map $f^{*}: \Omega_{G}^{*}(N) \rightarrow \Omega_{G}^{*}(M)$. It commutes with $d_{G}$ and hence produces a map in cohomology $f^{*}: H_{G}^{*}(N) \rightarrow H_{G}^{*}(M)$ which is a ring homomorphism. If $N$ is just a point, then $f^{*}$ gives a map from $S^{*}\left(\mathfrak{g}^{*}\right)$ to $H_{G}^{*}(M)$, which makes the equivariant cohomology of $M$ into an $S^{*}\left(\mathfrak{g}^{*}\right)$ module.

Let $i: F \rightarrow M$ be an invariant submanifold of $M$ which contains the fixed point set of the $G$ action. Then we have a very important fact, which is a part of ABBV abelian localization theorem (see [2]):

Theorem 2.1. The kernel of $i^{*}: H_{G}^{*}(M) \rightarrow H_{G}^{*}(F)$ is a torsion module and its support is in $\cup_{K} \mathfrak{k}^{c}$ where $K$ runs over the finite set of all isotropy groups not equal to $G$.

Remark 2.2. To remind the reader what the support of an $S^{*}\left(\mathfrak{g}^{*}\right)$ module $H$ is we repeat a classical definition which, for example, can be found in [3]. The support is the intersection of all hypersurfaces $V_{g}=\left\{u \in \mathfrak{g}^{c} \mid g(u)=0\right\}$, where $g$ runs through all polynomial on $\mathfrak{g}^{*}$ with $g H=0$ and $\mathfrak{g}^{c}$ stands for complexified Lie algebra.

Corollary 2.3. $H_{G}^{*}(M)$ is a torsion-free module over $S^{*}\left(\mathfrak{g}^{*}\right)$ if and only if $i^{*}$ : $H_{G}^{*}(M) \rightarrow H_{G}^{*}(F)$ is injective for any submanifold $F$ containing the fixed point set of the $G$ action.

Proof. By the localization theorem, the kernel of $i^{*}$ is a torsion module, but since $H_{G}^{*}(M)$ is torsion-free, the kernel of $i^{*}$ has to be trivial for any $F$. 
Conversely, take $F$ to be the fixed point set of the $G$ action, then $H_{G}^{*}(F)=$ $H^{*}(F) \otimes S^{*}\left(\mathfrak{g}^{*}\right)$ is a torsion-free module. Hence injectivity of $i^{*}$ implies that $H_{G}^{*}(M)$ is torsion-free.

Definition 2.4. Every manifold whose equivariant cohomology is torsion-free will be called equivariantly formal.

Remark 2.5. The equivariant formality has been studied in depth in [7], where $M$ is said to be equivariantly formal, if the spectral sequence for equivariant cohomology collapses at $E_{2}$. It was shown in [7] that this definition implies that the equivariant cohomology of $M$ is torsion-free. We believe that the opposite is also true, that is: our definition of the equivariant formality implies the collapse of the spectral sequence, but we do not have a proof of it.

A lot of sufficient conditions for the equivariant cohomology of $M$ to be torsion-free are given in [7]. For us, the main examples of equivariantly formal spaces will be symplectic manifolds with Hamiltonian torus actions (see [11]).

Let us also point out that the assumption that $M$ is equivariantly formal is crucial for most of our argument, since this property of $M$ allows to formally invert equivariant cohomology classes (see Lemma 2.8).

For an invariant map $f: M \rightarrow N$ between two oriented manifolds, we can also define the pushforward map in compactly supported cohomology $f_{*}$ : $H_{G, c}^{*+\operatorname{dim} M}(M) \rightarrow H_{G, c}^{*+\operatorname{dim} N}(N)$. ( $H_{G, c}^{*}$ stands for compactly supported cohomology). $f_{*}$ is always an $S^{*}\left(\mathfrak{g}^{*}\right)$ module homomorphism. The pushforward map to a point will often be denoted by integration. (For more details about equivariant pushforwards see [9]).

Assume that $Y$ is a compact oriented manifold with a $G$-action, $\pi: E \rightarrow Y$ is an oriented vector bundle of rank $n$ with a linear $G$-action on its fibers. Let the zero section be given by the inclusion $f: Y \rightarrow E$. We have the following fundamental property of pushforwards: for $a \in H_{G, c}^{*}(E)$ and $b \in H_{G}^{*}(Y)$

$$
\int_{E} a f_{*}(b)=\int_{Y} f^{*}(a) b .
$$

A Thom form $\tau$ is given by any closed form in $\Omega_{G, c}^{*}(E)$ with $[\tau]=f_{*} 1$. For any closed form $\nu \in \Omega_{G}^{*}(Y)$ we can say that $f_{*}[\nu]=\left[\pi^{*}(\nu) \tau\right]$. We also have the following identity:

$$
\int_{Y} \nu=\int_{E} \pi^{*}(\nu) \tau
$$

Remark 2.6. Notice that the above equation holds even if $\nu$ is not equivariantly closed; this is an important fact which will be used later.

Definition 2.7. For a nontrivial element $g \in S^{*}\left(\mathfrak{g}^{*}\right)$, we construct a new ring $S^{*}\left(\mathfrak{g}^{*}\right)_{g}$ of fractions $\left\{\frac{q}{g^{k}} ; q \in S^{*}\left(\mathfrak{g}^{*}\right)\right\}$ with $\frac{q}{g^{k}}=\frac{q^{\prime}}{g^{k^{\prime}}}$ if $q g^{k^{\prime}}=q^{\prime} g^{k}$. We say that the ring $S^{*}\left(\mathfrak{g}^{*}\right)_{g}$ is localized with respect to $g$. Since $H_{G}^{*}(Y)$ is an $S^{*}\left(\mathfrak{g}^{*}\right)$ module, 
we can localize it as well by constructing a new $S^{*}\left(\mathfrak{g}^{*}\right)_{g}$ module $H_{G}^{*}(Y)_{g}=$ $H_{G}^{*}(Y) \otimes_{S^{*}\left(\mathfrak{g}^{*}\right)} S^{*}\left(\mathfrak{g}^{*}\right)_{g}$. We call this operation inverting $g$. The elements of $H_{G}^{*}(Y)_{g}$ are called localized equivariant cohomology classes.

Assume that $E \rightarrow Y$ is a complex vector bundle on which $G$ acts by an action compatible with the action of $G$ on $Y$ and that $f: Y \rightarrow E$ is the inclusion map. The equivariant Euler class of $E$ is defined by $e(E)=f^{*} f_{*}(1)$. The following lemma is a crucial fact about equivariant Euler classes of complex vector bundles.

Lemma 2.8. Given a subgroup $S^{1} \subset G$ assume that the fixed point set of the $S^{1}$ action on $E$ is precisely $Y$. Then after inverting a finite number of elements in $S^{*}\left(\mathfrak{g}^{*}\right)$, the Euler class $e(E)$ becomes invertible.

Proof. We have the map $p^{*}: S^{*}\left(\mathfrak{g}^{*}\right) \rightarrow H_{G}^{*}(Y)$ coming from the projection $p: Y \rightarrow$ point. It is enough to find $b \in H_{G}^{*}(Y)$ and $g \in S^{*}\left(\mathfrak{g}^{*}\right)$ such that $e(E) b=p^{*}(g)$, since then $e(E)$ becomes invertible after localizing with respect to $g$. It was proved by Atiyah and Bott in [2] that the Euler class $e(E)$ is not a zero divisor when the fixed point set of the $S^{1}$ action is exactly $M$. Hence the Thom exact sequence for $E$ splits into short exact sequences

$$
0 \rightarrow H_{G}^{*-n}(Y) \stackrel{\cdot e(E)}{\rightarrow} H_{G}^{*}(Y) \stackrel{\pi^{*}}{\rightarrow} H_{G}^{*}(S E) \rightarrow 0,
$$

where $\pi: S E \rightarrow Y$ is the sphere bundle of $E$ for some invariant metric on $E$. So, our goal is to prove that the map $\pi^{*}: \operatorname{im}\left(p^{*}\right) \rightarrow H_{G}^{*}(S E)$ has a nontrivial kernel.

Choose a point $x \in Y$ such that its isotropy group $G_{x}$ has maximal rank. The equivariant cohomology of the orbit $G \cdot x$ through $x$ is equal to $H_{G_{x}}^{*}$ (point). Given the inclusion $G \cdot x \rightarrow M$ the map $S^{*}\left(\mathfrak{g}^{*}\right) \stackrel{p^{*}}{\rightarrow} H_{G}^{*}(Y) \rightarrow H_{G}^{*}(G \cdot x)$ is onto. Hence $\operatorname{dim}_{k}\left(\operatorname{im}\left(p^{*}\right)\right) \geq \operatorname{dim} H_{G_{x}}^{k}$ (point) $=\operatorname{dim} S^{k}\left(\mathfrak{g}_{x}^{*}\right)$, where $\mathfrak{g}_{x}$ is the Lie algebra of $G_{x}$. On the other hand, we will prove that if $H=G_{x} / S^{1}$ and $\mathfrak{h}$ is its Lie algebra then $\operatorname{dim} H_{G}^{k}(S E) \leq C \operatorname{dim} S^{k}\left(\mathfrak{h}^{*}\right)$ for some constant $C$. Hence, to show that $\pi^{*}: \operatorname{im}\left(p^{*}\right) \rightarrow H_{G}^{*}(S E)$ has a nontrivial kernel, it is enough to prove that as $k$ grows, the dimension of $S^{k}\left(\mathfrak{g}_{x}^{*}\right)$ grows faster than the dimension of $S^{k}\left(\mathfrak{h}^{*}\right)$. But $\operatorname{dim} S^{k}\left(\mathfrak{g}_{x}^{*}\right)=\left(\begin{array}{c}\operatorname{dim} G_{x}+k-1 \\ k\end{array}\right)$ and $\operatorname{dim} S^{k}\left(\mathfrak{h}^{*}\right)=\left(\begin{array}{c}\operatorname{dim} H+k-1 \\ k\end{array}\right)$, therefore $\frac{\operatorname{dim} S^{k}\left(\mathfrak{g}_{x}^{*}\right)}{\operatorname{dim} S^{k}\left(\mathfrak{h}^{*}\right)}=$ $\frac{\operatorname{dim} G_{x}+k-1}{\operatorname{dim} G_{x}-1} \rightarrow \infty$ as $k \rightarrow \infty$. We run into a problem when $\operatorname{dim} G_{x}=1$ and we cannot divide by $\operatorname{dim} G_{x}-1=0$, but then it easy to see that $H_{G}^{*}(S E)$ is finite dimensional, while $\operatorname{im}\left(p^{*}\right)$ is not.

It is only left to prove that $\operatorname{dim} H_{G}^{k}(S E) \leq C \operatorname{dim} S^{k}\left(\mathfrak{h}^{*}\right)$. The maximal rank of the isotropy group for the action of $G$ on $S E$ is at most $\operatorname{dim} G_{x}-1$. Hence we can apply localization theorem 2.1 to show that $H_{G}^{*}(S E)$ is supported in a union of subspaces $\cup_{K} \mathfrak{k}^{c}$ of $\mathfrak{g}^{*}$, where $K$ runs through a finite number of isotropy subgroups, each of dimension at most $\operatorname{dim} H$. Combining this with the fact that $H_{G}^{*}(S E)$ is finitely generated as $S^{*}\left(\mathfrak{g}^{*}\right)$ module proves that $\operatorname{dim} H_{G}^{k}(S E) \leq$ $C \operatorname{dim} S^{k}\left(\mathfrak{h}^{*}\right)$ for some large constant $C$. Hence we have proved the lemma. 
Given equation (1) and Lemma 2.8 we can, following the ideas of Guillemin and Sternberg in [9], get the following equation for $a \in H_{G, c}^{*}(E)$ :

$$
\int_{E} a=\int_{Y} \frac{f^{*}(a)}{e(E)}
$$

Indeed, let the cohomology class $a$ be supported in some neighborhood $U$ of $M$; then it is easy to see that $\tau$ is cohomologous to $\pi^{*} f^{*} \tau$ on $U$. Hence for $b$ found in the proof of the Lemma 2.8, we use equation (1)

$$
\begin{aligned}
\int_{Y} f^{*}(a) b & =\int_{E} a f_{*}(b)=\int_{E} a \pi^{*}(b)[\tau] \\
& =\int_{E} a \pi^{*}(b e(E))=\int_{E} a \pi^{*} p^{*}(g) .
\end{aligned}
$$

After inverting $g,(3)$ holds with $\frac{1}{e(E)}=\frac{b}{p^{*}(g)}$.

The above computation allows us to use the setting of Lemma 2.8 and prove the following.

Lemma 2.9. For a $G$-invariant Hermitian metric on $E$ let $S E$ denote the unit sphere bundle over $Y$. For a closed form $\lambda \in \Omega_{G}^{*}(Y)$ of degree less than $\operatorname{dim} E$,

$$
\left.\int_{S E} \sigma\right|_{S E}=-\int_{Y} \frac{\left[f^{*} \omega\right]}{e(E)}
$$

where $\sigma$ is any (possibly localized) equivariant form defined outside of $Y$ with $d_{G} \sigma=\omega$ on $E-Y$.

Proof. Denote by $D E$ the radius 1 disc bundle of $E$. Let $\psi$ be an invariant bump function on $E$ which is 0 outside of $D E$ and is equal to 1 in the neighborhood of $Y$. Apply equivariant Stokes' theorem on $D E$ :

$$
\left.\int_{S E} \sigma\right|_{S E}=\int_{D E} d_{G}((1-\psi) \sigma)=\int_{D E} \omega-\int_{D E-Y} d_{G}(\psi \sigma) .
$$

Notice that the second integral is over $D E-Y$, since $\sigma$ is not defined on $Y$, but since $\psi=1$ in a neighborhood of $Y$, we know $d_{G}(\psi \sigma)=\omega$ in this neighborhood of $Y$. Hence $d_{G}(\psi \sigma)$ could be extended to a form $\tilde{\omega}$ with $\omega=\tilde{\omega}$ in some neighborhood of $Y$. At the same time, $\int_{D E} \omega=0$, since $\operatorname{deg} \omega<\operatorname{dim} E$. Therefore,

$$
\left.\int_{S E} \sigma\right|_{S E}=-\int_{E} \tilde{\omega} .
$$

But $\tilde{\omega}$ is a compactly supported closed form and equation (3) implies

$$
\left.\int_{S E} \sigma\right|_{S E}=-\int_{Y} \frac{f^{*} \tilde{\omega}}{e(E)}=-\int_{Y} \frac{f^{*} \omega}{e(E)} .
$$




\section{Circle action}

Assume that we have a compact abelian group $G$ together with a circle $S^{1} \subset G$ inside. Let $M$ be a compact connected oriented manifold with a $G$ action. Denote by $i_{j}: F_{j} \rightarrow M$ the connected components of the fixed point set of the circle action and by $N F_{j}$ the normal bundles of $F_{j}$ 's. Notice that because of the circle action, all the $N F_{j}$ can be given a complex structure and, in particular, all $F_{j}$ are orientable and of even codimension.

Theorem 3.1. For a possibly localized equivariantly closed form $\omega$ on $M$

$$
\int_{M} \omega=\sum_{F_{j}} \int_{F_{j}} \frac{i_{j}^{*}[\omega]}{e\left(N F_{j}\right)},
$$

where $e\left(N F_{j}\right)$ are the equivariant Euler classes of the normal bundles $N F_{j}$ 's.

We omit the proof of this theorem, since it has been proven by many authors. Let just point out that there are two minor differences between Theorem 3.1 and the standard statement of the ABBV theorem. First of all, the form $\omega$ could be localized in our case. Secondly, we consider the fixed points of the subcircle action rather than fixed points of the $G$ action. Even more generally, manifolds $F_{j}$ can be any connected components of the fixed point set of any subtori actions which contain the fixed points of the $G$ action (even more general statement of the ABBV theorem can be found in in [4], Theorem 7.13).

Next, assume that $M$ is equivariantly formal and we are given an invariant oriented connected submanifold $W$ and the inclusion map $I: W \rightarrow M$. We assume that $S^{1}$ acts freely on $W$. Let $X$ be the manifold $W / S^{1}$. The orientation on $W$ together with an orientation on $S^{1}$ induces an orientation on $X$. We always have the Cartan isomorphism $c: H_{G}^{*}(W) \rightarrow H_{G / S^{1}}^{*}(X)$. We will be concerned with understanding the following composition of maps $\kappa: H_{G}^{*}(M) \stackrel{I^{*}}{\rightarrow} H_{G}^{*}(W) \stackrel{c}{\rightarrow}$ $H_{G / S^{1}}^{*}(X)$. If $M$ is a Hamiltonian $S^{1}$-manifold and $W$ is the zero level of a moment map, then $\kappa$ is just the Kirwan map and, since we assume $S^{1}$ acts freely on $W$, zero is a regular value of the moment map, and $\kappa$ is surjective.

A closed form $\alpha \in \Omega_{G / S^{1}}^{*}(X)$ lifts to an equivariant cohomology class $c^{-1}([\alpha])$ on $W$. Choose a small invariant neighborhood $U$ of $W$ which does not intersect the fixed point set of $S^{1}$. Then the equivariant pushforward $I_{*}$ factors through the map to compactly supported cohomology of $U: H_{G}^{*-\operatorname{codim} W}(W) \rightarrow$ $H_{G, c}^{*}(U) \rightarrow H_{G}^{*}(M)$. Let $\beta$ be an equivariantly closed form which represents $I_{*} c^{-1}([\alpha])$ in $\Omega_{G, c}^{*}(U)$. Clearly, $\beta$ is in $\Omega_{G}^{*}(M)$. Since $U$ does not intersect the fixed point set, the support of $\beta$ does not intersect it either and by Corollary 2.3 we can choose an equivariant form $\mu$ such that $d_{G} \mu=\beta$. We will call $\mu$ a transgression form of $\alpha$. Let us remark that the definition of transgression form is the only place in the paper where we use the equivariant formality of $M$. Hence the results of this paper would be always true as long as we can define $\mu$.

Before proving our main theorem, let us explain why such a result should hold. For a closed form $\omega \in H_{G}^{*}(M)$ of degree less than $\operatorname{dim} W-\operatorname{deg} \alpha+1$, 
consider the following sum:

$$
S(\omega \mu)=\sum_{F_{j}} \int_{F_{j}} \frac{\left[i_{j}^{*}(\omega \mu)\right]}{e\left(N F_{j}\right)} .
$$

Observe that $i_{j}^{*}(\omega \mu)$ are indeed closed forms, since the support of $d_{G} \mu$ lies outside of the $F_{j}$ 's.

It is easy to see that the above sum does not depend on the choice of $\beta$ and $\mu$. Indeed, if we have other $\beta^{\prime}$ and $\mu^{\prime}$ with $d_{G} \mu^{\prime}=\beta^{\prime}$, then we can choose a form $\nu$ in $\Omega_{G, c}^{*}(U)$ such that $d_{G} \nu=\beta-\beta^{\prime}$. Hence $\mu-\mu^{\prime}=\nu+\gamma$, where $\gamma$ is an equivariantly closed form on $M$ and

$$
S(\omega \mu)-S\left(\omega \mu^{\prime}\right)=S(\omega \nu)+S(\omega \gamma) .
$$

But $S(\omega \nu)=0$, since the support of $\nu$ does not intersect the fixed point set. Moreover, $\omega \gamma$ is a closed form on $M$ and by Theorem 3.1,

$$
S(\omega \gamma)=\int_{M}[\omega \gamma] .
$$

But $\omega \gamma$ has degree less than dimension of $M$ which implies that the above integral is equal to 0 . Therefore the sum (4) is the same for $\mu$ and $\mu^{\prime}$ and it is very easy to conclude that it should depend only on the cohomology classes $[\alpha]$ and $[\omega]$. Theorem 3.2 gives an explicit expression of (4) in terms of $[\alpha]$ and $[\omega]$.

To state Theorem 3.2, recall that we assume that $M$ is equivariantly formal, $W$ is an invariant oriented connected submanifold on $M$, on which $S^{1}$ acts freely, $W / S^{1}=X$. Let $e(W)$ be the Euler class of the associated complex line bundle $L_{W}=W \times_{S^{1}} \mathbb{C}$ over $X$, where $S^{1}$ acts on $\mathbb{C}$ with weight 1 . For an equivariantly closed form $\alpha \in \Omega_{G / S^{1}}^{*}(X)$, let $\mu$ denote its transgression form. The map $G \rightarrow G / S^{1}$ induces the inclusion $H_{G / S^{1}}^{*}(X) \rightarrow H_{G}^{*}(X)$, which allows us to think of classes in $H_{G / S^{1}}^{*}(X)$ as classes in $H_{G}^{*}(X)$. This setting leads to the statement and the proof of our main theorem.

Theorem 3.2. For any equivariantly closed forms $\alpha \in \Omega_{G / S^{1}}^{*}(X)$ and $\omega \in$ $\Omega_{G}^{*}(M)$ such that $\operatorname{deg} \alpha+\operatorname{deg} \omega \leq \operatorname{dim} W$

$$
(-1)^{\operatorname{deg} \omega+1} \int_{X} \frac{\kappa([\omega])[\alpha]}{e(W)}=\sum_{F_{j}} \int_{F_{j}} \frac{\left[i_{j}^{*}(\omega \mu)\right]}{e\left(N F_{j}\right)},
$$

where $e(W)$ is the equivariant Euler class of the circle bundle $W \rightarrow X$ and $e\left(N F_{j}\right)$ are the equivariant Euler classes of the normal bundles $N F_{j}$ of $F_{j}$.

Proof. Consider a small invariant tubular neighborhood $\pi: U \rightarrow W$ of $W$ which does not intersect any of $F_{j}$ 's. By Theorem 2.1, we can find a form $\nu$ in some localized ring such that $d_{G} \nu=\omega$ on $M-\left(\cup_{j} F_{j}\right)$.

First of all, we would like to show that without loss of generality we can assume that

$$
\nu=\pi^{*} I^{*} \nu \text { on } U
$$


Choose another invariant neighborhood $\tilde{\pi}: \tilde{U} \rightarrow W$ such that it contains the closure of $U,\left.\tilde{\pi}\right|_{U}=\pi$ and $\tilde{U}$ does not intersect the fixed point set of the $S^{1}$ action. The form $\omega-\tilde{\pi}^{*} I^{*} \omega$ is cohomologous to zero on $\tilde{U}$, so we can choose a form $\gamma$ with $d_{G} \gamma=\omega-\tilde{\pi}^{*} I^{*} \omega$ on $\tilde{U}$. Choose a bump function $\psi$ with $\psi=1$ on $U$ and $\psi=0$ outside $\tilde{U}$. Then substituting $\omega$ with $\omega-d_{G}(\psi \gamma)$ will make $\omega=\pi^{*} I^{*} \omega$ on $U$ and at the same time will not change $I^{*} \omega$ or any $i_{j}^{*} \omega$. After we showed $\omega=\pi^{*} I^{*} \omega$ on $U$, we apply a similar trick to $\nu$.

Without loss of generality, we can assume $\omega=\tilde{\pi}^{*} I^{*} \omega$ on $\tilde{U}$ and hence $\nu$ will satisfy $d_{G}\left(\nu-\tilde{\pi}^{*} I^{*} \nu\right)=0$ on $\tilde{U}$. Thus, after some localization, we can find a localized form $\eta$ with $\nu-\tilde{\pi}^{*} I^{*} \nu=d_{G} \eta$ on $\tilde{U}$ by Theorem 2.1. We can substitute $\nu$ with $\nu-d_{G}(\psi \eta)$ without changing $d_{G} \nu$ and after this substitution $\nu$ will satisfy $(5)$.

Now consider the form $\omega \mu+(-1)^{\operatorname{deg} \nu} \nu \beta$. This form is equivariantly closed and $i_{j}^{*}\left(\omega \mu+(-1)^{\operatorname{deg} \nu} \nu \beta\right)=i_{j}^{*}(\omega \mu)$, since $\beta$ is supported outside of $F_{j}$ 's. Apply Theorem 3.1 to this form:

$$
\int_{M}\left(\omega \mu+(-1)^{\operatorname{deg} \nu} \nu \beta\right)=\sum_{F_{j}} \int_{F_{j}} \frac{\left[i_{j}^{*}(\omega \mu)\right]}{e\left(N F_{j}\right)} .
$$

Notice that $\operatorname{deg}(\omega \mu)<\operatorname{dim} M$, moreover, $\omega \mu$ is an honest (not localized) form, thus:

$$
\int_{M} \omega \mu=0
$$

Choose a closed form $\theta \in \Omega_{G}^{*}(W)$ with $[\theta]=c^{-1}([\alpha])$. Similarly to equation (1) we would like to prove:

$$
\int_{U} \nu \beta=\int_{W} I^{*}(\nu) \theta .
$$

This would be true if $\nu \beta=\pi^{*}\left(I^{*}(\nu) \theta\right) \tau$ on $U$ so that we could use equation (2). But we know that we can choose $\beta=\pi^{*}(\theta) \tau$, since the sum (4) does not depend on the choice of $\beta$. Combining it with equation (5), we see that we could assume from the very beginning that $\nu \beta=\pi^{*}\left(I^{*}(\nu) \theta\right) \tau$. Hence (8) holds.

Finally, we will deal with computing $\int_{W} I^{*}(\nu) \theta$. Consider a weight one action of $S^{1}$ on $\mathbb{C}$ and the space $L_{W}=W \times{ }_{S^{1}} \mathbb{C}$ constructed by taking the quotient of $W \times \mathbb{C}$ by the diagonal circle action. It is a complex line bundle over $X$ together with a $G$ action coming from the action on $W$. Since $S^{1}$ fixes $X$ and does not fix any other point in $L_{W}$, Lemma 2.8 implies that the equivariant Euler class $e\left(L_{W}\right)=e(W)$ is invertible in some localized ring. Using Lemma 2.9 we will prove that

$$
\int_{W} I^{*}(\nu) \theta=\int_{X} \frac{\kappa([\omega])[\alpha]}{e\left(L_{W}\right)} .
$$

A combination of the equations (6), (7), (8), and (9) proves the theorem. Hence, it is left to carry out the computation which establishes (9). 
Given a Hermitian metric on $\mathbb{C}$, we can identify the radius 1 circle bundle $S L_{W} \subset L_{W}$ with $W$. The equivariant form $I^{*}(\omega) \theta \otimes 1$ sits inside $\Omega_{G \times S^{1}}^{*}(W \times \mathbb{C})$. Since $H_{G \times S^{1}}^{*}(W \times \mathbb{C})=H_{G}^{*}\left(L_{W}\right)$, we can choose a closed form $\lambda \in \Omega_{G}^{*}\left(L_{W}\right)$ with $\left[I^{*}(\omega) \theta \otimes 1\right]=[\lambda]$, also $\left.[\lambda]\right|_{X}$ will be exactly $\kappa([\omega])[\alpha]$. Moreover, since $d_{G}\left(\left(I^{*}(\nu) \theta\right) \otimes 1\right)=I^{*}(\omega) \theta \otimes 1$ on $W$, we can apply Lemma 2.9 with $\left.\sigma\right|_{W}=I^{*}(\nu) \theta$ to prove

$$
\int_{W} I^{*}(\nu) \theta=\int_{X} \frac{\left.[\lambda]\right|_{X}}{e\left(L_{W}\right)}=\int_{X} \frac{\kappa([\omega])[\alpha]}{e(W)} .
$$

Notice that there is no minus sign in the above equation, since the orientations on $W$ and $S E$ are opposite. This finishes the proof of (9) and the theorem.

Remark 3.3. If the map $\kappa$ is surjective, then $[\alpha]=\kappa([\gamma])$ for some $\gamma$. Taking 1 instead of $\alpha$ and $\omega \gamma$ instead of $\omega$ shows that adding $[\alpha]$ into the statement of the theorem does not bring any new information. But in the next section we give an example where $\kappa$ is not surjective and the presence of $\alpha$ is important for successful application of the theorem.

\section{Residues and other applications}

In this section we define residues, formulate another localization theorem using residues, and show how to deduce a special case of the Jeffrey-Kirwan localization theorem from our results. We also provide two non-symplectic examples and, at the end of the section, we show how our theorems work in the orbifold case.

To define residues we will need a bit more information about equivariant cohomology. As in Lemma 2.8, consider a complex vector bundle $\pi: E \rightarrow Y$ with a linear $G$ action on the fibers, such that the fixed point set of the subcircle $S^{1} \subset G$ action is exactly $Y$. We again call $H=G / S^{1}$ and we have a natural inclusion $\mathfrak{h}^{*} \rightarrow \mathfrak{g}^{*}$ which induces the injection $H_{H}^{*}(Y) \rightarrow H_{G}^{*}(Y)$ for the reason given below.

Let $x_{1}$ be a vector in $\mathfrak{g}$, which is an integer basis of the tangent space to $S^{1}$. Complete $x_{1}$ to a basis $x_{a}$ of $\mathfrak{g}$. Let $u_{a}$ be the dual basis to $x_{a}$ and let $u=u_{1}$; then we can say that $S^{*}\left(\mathfrak{g}^{*}\right)=S^{*}\left(\mathfrak{h}^{*}\right) \otimes \mathbb{C}[u]$, in particular $\Omega_{G}^{*}(Y)=\Omega_{H}^{*}(Y) \otimes \mathbb{C}[u]$. Elements $x_{2}, \ldots, x_{n}$ produce a basis in $\mathfrak{h}$ and since the action of $S^{1}$ on $Y$ is trivial, it is easy to see $d_{G}=d_{H}$, which implies $H_{G}^{*}(Y)=H_{H}^{*}(Y) \otimes \mathbb{C}[u]$, and in particular, $H_{H}^{*}(Y)$ is injected into $H_{G}^{*}(Y)$.

Assume that $E$ splits as a sum of invariant complex line bundles $L_{1} \oplus \ldots \oplus L_{k}$ (we can do that without loss of generality by applying the equivariant splitting principle). $S^{1}$ acts on $L_{i}$ 's with some weights $m_{i}$ 's, and we can prove the following easy lemma.

Lemma 4.1. $e\left(L_{i}\right)=m_{i} u+h_{i}$, where $h_{i} \in H_{H}^{*}(Y)$.

Proof. Since the degree of $e\left(L_{i}\right)$ is equal to 2, we can say that $e\left(L_{i}\right)=c u+h_{i}$ where $c$ is some constant and $h_{i} \in H_{H}^{2}(Y)$. From the inclusion $S^{1} \rightarrow G$ we derive the map in equivariant cohomology $H_{G}^{*}(Y) \rightarrow H_{S^{1}}^{*}(Y)$. Moreover, under 
this map $e\left(L_{i}\right)$ goes to $e_{S^{1}}\left(L_{i}\right)=c u+h$, where $e_{S^{1}}\left(L_{i}\right)$ is the equivariant Euler class with respect to the $S^{1}$ action and $h \in H^{2}(Y)$. But it is well known that $e_{S^{1}}\left(L_{i}\right)=m_{i} u+c_{1}\left(L_{i}\right)$, where $c_{1}\left(L_{i}\right)$ is the first Chern class of $L_{i}$. This finishes the proof of the lemma.

We are now ready to define the residue of $\frac{a}{e(E)}$ for a cohomology class $a=$ $\sum_{j=0}^{m} g_{j} u^{j} \in H_{G}^{*}(Y)$ with $g_{j} \in H_{H}^{*}(Y)$. We will basically repeat the definition of residues given by Guillemin and Kalkman in [8].

Expand $\frac{a}{e(E)}$ in a possibly infinite formal Laurent series

$$
\begin{aligned}
\frac{a}{e(E)} & =\left(\sum_{j=0}^{m} g_{j} u^{j}\right)\left(\prod_{i} e\left(L_{i}\right)^{-1}\right)=\left(\sum_{j=0}^{m} g_{j} u^{j}\right)\left(\prod_{i}\left(m_{i} u\right)^{-1} \frac{1}{1+\frac{h_{i}}{m_{i} u}}\right) \\
& =\left(\sum_{j=0}^{m} g_{j} u^{j}\right)\left(\prod_{i}\left(m_{i} u\right)^{-1}\left(1-\frac{h_{i}}{m_{i} u}+\frac{h_{i}^{2}}{\left(m_{i} u\right)^{2}}-\ldots .\right)\right)=\sum_{k<m} b_{k} u^{k}
\end{aligned}
$$

where $b_{k}$ 's are expressed in terms of $g_{j}$ 's, $h_{i}$ 's and $m_{i}$ 's.

Definition 4.2. $\operatorname{Res}\left(\frac{a}{e(E)}\right)=b_{-1}$.

Let us understand how the defined residue depends on the choice of the original basis $x_{a}$. We have two choices of $x_{1}$, and the substitution of $x_{1}$ to its opposite $-x_{1}$ in the definition will change Res to - Res. A different choice of $x_{2}, \ldots, x_{n}$ will make $u$ equal to $u+h$, where $h \in H_{H}^{2}(Y)$. This change will not alter our residue for the reasons given in [8]. That is, for $k \geq 0$ the polynomials $b_{k}(u+h)^{k}$ will not provide any terms with negative powers of $u$; for $k \leq-1$ the terms of $b_{k}(u+h)^{k}$ will change only lower than degree -1 terms. Hence $b_{-1}$ does not depend on the choice of $x_{2}, \ldots, x_{n}$.

Notice that Definition 4.2 makes sense for a possibly localized cohomology class as long as we can construct an infinite Laurent series that unambiguously defines the coefficient in front of $u^{-1}$. In particular, we can define the residue of any nonlocalized equivariant cohomology class $a$ by $\operatorname{Res}(a)=0$. Moreover, $\int_{Y} \frac{a}{e(E)}$ has a Laurent series in $S^{*}\left(\mathfrak{g}^{*}\right)$, which can be constructed with the same argument presented above. Hence the residue of $\int_{Y} \frac{a}{e(E)}$ is well defined, and Res will always commute with the integration

$$
\operatorname{Res} \int_{Y} \frac{a}{e(E)}=\int_{Y} \operatorname{Res} \frac{a}{e(E)} .
$$

We are ready to state our second theorem. Notice that in this theorem we do not make any restriction on the degrees of $\omega$ and $\alpha$. Also, the right choice of the sign of the residue will be specified during the proof of the theorem.

Theorem 4.3. For any equivariantly closed forms $\alpha \in \Omega_{G / S^{1}}^{*}(X)$ and $\omega \in$ $\Omega_{G}^{*}(M)$

$$
(-1)^{\operatorname{deg} \omega} \int_{X} \kappa([\omega])[\alpha]=\sum_{F_{j}} \int_{F_{j}} \operatorname{Res} \frac{\left[i_{j}^{*}(\omega \mu)\right]}{e\left(N F_{j}\right)},
$$


where $e\left(N F_{j}\right)$ are the equivariant Euler classes of the normal bundles $N F_{j}$.

Proof. Here the proof of Theorem 3.2 could be repeated word-for-word . The only difference would be that in a couple of places in the proofs of Theorem 3.2 and Lemma 2.9 we would not be able to argue that some integrals are zeros because we are integrating nonlocalized equivariant forms of small enough degrees. But we will be able to prove the following:

$$
(-1)^{\operatorname{deg} \omega+1} \int_{X} \frac{\kappa([\omega])[\alpha]}{e(W)}=\sum_{F_{j}} \int_{F_{j}} \frac{\left[i_{j}^{*}(\omega \mu)\right]}{e\left(N F_{j}\right)}+O,
$$

where $O$ is a remainder given by the integrals of nonlocalized forms. Since Res is linear and commutes with integration and the residue of any nonlocalized form is zero we can conclude

$$
(-1)^{\operatorname{deg} \omega+1} \int_{X} \operatorname{Res} \frac{\kappa([\omega])[\alpha]}{e(W)}=\sum_{F_{j}} \int_{F_{j}} \operatorname{Res} \frac{\left[i_{j}^{*}(\omega \mu)\right]}{e\left(N F_{j}\right)} .
$$

Hence, it is only left to show that

$$
\operatorname{Res} \frac{\kappa([\omega])[\alpha]}{e(W)}=-\kappa([\omega])[\alpha] .
$$

We know that the weight of the $S^{1}$ action on $L_{W}$ is 1 ; hence $e(W)=u+h$ by Lemma 4.1. Moreover, $\kappa([\omega])[\alpha] \in H_{G / S^{1}}^{*}(X)$. Hence after we choose a sign of the residue that is compatible with $u$, the equation (10) will hold.

4.4. Symplectic Case. Let us now explain how the above result implies Kalkman's circle localization theorem (see [11]), which is a special case of the JeffreyKirwan theorem. Let $M$ be an $S^{1}$ Hamiltonian space with a moment map $\phi: M \rightarrow \mathbb{R}$. Assume 0 is a regular value of the moment map and $W$ is the zero level set $\phi^{-1}(0)$ on which $S^{1}$ acts freely, let $\alpha=1$. We can choose $U$ to be the preimage of a small interval $(-\epsilon, \epsilon) \subset \mathbb{R}$. Then $U$ could be identified with $(-\epsilon, \epsilon) \times W$, since the normal bundle of $W$ is trivial. Pick a bump function $\rho$ on $\mathbb{R}$ that is 0 outside $(-\epsilon, \epsilon)$ and $\int_{\mathbb{R}} \rho d \phi=1$. Then we can assume $\beta=\tau=\rho d \phi$ on $U$ and extend it by 0 to the rest of $M$. The form $\mu$ will be merely the function

$$
\mu\left(t_{0}\right)=\int_{-\epsilon}^{t_{0}} \rho d \phi, \text { for } t_{0} \in(-\epsilon, \epsilon)
$$

Even though $\mu$ is defined only on $U$, we can extend it to any $x \in M$ by $\mu(x)=0$ if $\phi(x)<-\epsilon$ and $\mu(x)=1$ if $\phi(x)>\epsilon$. For any $\omega \in \Omega_{G}^{\operatorname{dim} M-2}(M)$, Theorem 4.3 reduces to

$$
\int_{X} \kappa([\omega])=\sum_{j} \int_{F_{j}} \operatorname{Res} \frac{\left[i_{j}^{*}(\omega \mu)\right]}{e\left(N F_{i}\right)}=\sum_{\phi\left(F_{j}\right)>0} \int_{F_{j}} \operatorname{Res} \frac{\left[i_{j}^{*}(\omega)\right]}{e\left(N F_{i}\right)},
$$

which is exactly the statement of the Jeffrey-Kirwan theorem given in [11]. If we apply Theorem 3.2 to this setting, we will get a formula, which was also obtained by Lerman in [14] using symplectic cuts. 
4.5. Examples. Let us now present two examples that illustrate the use of our theorems in the absence of any symplectic structure.

Example 4.6. Consider a sphere

$$
M=S^{2 n}=\left\{\left(r, z_{1}, \ldots, z_{n}\right) \in \mathbb{R} \times \mathbb{C}^{n} ; r^{2}+\left|z_{1}\right|^{2}+\ldots+\left|z_{n}\right|^{2}=1\right\}
$$

together with the circle action given by $\theta\left(r, z_{1}, \ldots ., z_{n}\right)=\left(r, \theta z_{1}, \ldots, \theta z_{n}\right)$ with $\theta \in S^{1} \subset \mathbb{C}$. Its equivariant cohomology injects into the equivariant cohomology of the north and south poles: $i^{*}: H_{S^{1}}^{*}\left(S^{2 n}\right) \rightarrow H_{S^{1}}^{*}\left(p_{n}\right) \oplus H_{S^{1}}^{*}\left(p_{s}\right)=\mathbb{C}[u] \oplus \mathbb{C}[v]$, where $p_{n}=(1,0, \ldots, 0)$ and $p_{s}=(-1,0, \ldots, 0)$. It is easy to understand the image of the above map:

$$
i^{*} H_{S^{1}}^{k}\left(S^{2 n}\right)= \begin{cases}\text { multiples of }\left(u^{k}+v^{k}\right), & \text { if } k<2 n-1 \\ \mathbb{C}^{k}[u] \oplus \mathbb{C}^{k}[v], & \text { if } k>2 n-1 .\end{cases}
$$

Consider a subsphere $W=S^{2 k-1}$ consisting of points $\left\{\left(0, z_{1}, \ldots, z_{k}, 0, \ldots, 0\right) \in\right.$ $\left.S^{2 n}\right\} . S^{2 k-1} / S^{1}$ is the projective space $X=\mathbb{P}^{k-1}$. Take $\alpha=1$ on $\mathbb{P}^{k-1}$, then $\beta$ is equal to a Thom form of $S^{2 k-1}$ and we denote by $\mu_{k}$ a transgression form for this $\beta$. Let $H^{2 k}=\left\{\left(r, z_{1}, \ldots, z_{k}, 0, \ldots, 0\right) \in S^{2 n}\right\}$, and denote by $\tau_{k}$ a Thom form for $H^{2 k}$. Then we can choose $\mu_{k}$ so that

$$
\mu_{k}=\tau_{k} \mu_{n},
$$

where $\mu_{n}$ is a transgression form for the equator $S^{2 n-1} \subset S^{2 n}$. Moreover, $\mu_{n}$ could be constructed in the same way in which we produced the transgression form in the symplectic case, with $r$ substituted for $\phi$. The map $r$ serves as an abstract moment map. (See [6] for definition of abstract moment maps and further references.) To apply our theorem, we have merely to understand the restriction of $\mu_{k}$ to the fixed point set. Since $\left.\mu_{n}\right|_{p_{s}}=0$ and $\left.\mu_{n}\right|_{p_{n}}=1$ we can conclude that $\mu_{k}$ is zero if restricted to the south pole and $\left.\mu_{k}\right|_{p_{n}}=\left.\tau_{k}\right|_{p_{n}}=u^{n-k}$. Hence our localization theorem states that for $[\omega]=u^{\ell}+v^{\ell}$ with $\ell<k$

$$
-\int_{\mathbb{P}^{k-1}} \frac{\kappa[\omega]}{u+c}=\int_{p_{n}} \frac{u^{n-k} \cdot\left[\left.\omega\right|_{p_{n}}\right]}{u^{n}}=\frac{u^{\ell}}{u^{k}},
$$

where $c$ is the Chern class of the circle bundle $S^{2 k-1} \rightarrow \mathbb{P}^{k-1}$. Hence, we have $\kappa\left(u^{\ell}+v^{\ell}\right)=(-1)^{\ell} c^{\ell}$ for $\ell<k$.

Example 4.7. In the previous example the Kirwan map was surjective which is always the case in the symplectic setting. Let us now give an example where the Kirwan map is no longer surjective. In this example the addition of the form $\alpha$ into the statements of our theorems becomes very important.

As before $M$ is going to be the $2 n$ sphere with the same circle action. But we will change the submanifold $W$ to a product of two spheres $S^{2 k-1} \times S^{2 m-1}$ :

$W=\left\{\left(0, z_{1}, \ldots, z_{k+m}, 0, \ldots, 0\right) \in S^{2 n} ;\left|z_{1}\right|^{2}+\ldots+\left|z_{k}\right|^{2}=\left|z_{k+1}\right|^{2}+\ldots+\left|z_{k+m}\right|^{2}\right\}$ with $k<m$. Then it is not difficult to compute the cohomology of $X=W / S^{1}$. It is generated by two elements $c$ and $t$ of degrees 2 and $2 m-1$ respectively with the following relations:

$$
c^{k}=0, \text { and } t^{2}=0
$$


Moreover, $c$ is the Chern class of the circle bundle $W \rightarrow X$ and $t$ is the Thom class of the manifold $V / S^{1}$, where $V$ is the $2 k-1$ sphere

$$
V=\left\{\left(0, z_{1}, \ldots z_{k}, z_{1}, \ldots, z_{k}, 0, \ldots 0\right) \in S^{2 n}\right\} \subset W .
$$

To apply our theorem we take $[\alpha]=t$ so that $\beta$ is a Thom form of $V$ inside $S^{2 n}$. Similarly to the argument in the previous example, we can conclude that $\mu$ restricts to 0 at $p_{s}$, and it restricts to $u^{n-k}$ at $p_{n}$, since in the neighborhood of $p_{n}$ we can assume $\mu$ is equal to a Thom form of the sphere $H=\left\{\left(r, z_{1}, \ldots z_{k}, z_{1}, \ldots, z_{k}, 0, \ldots 0\right) \in S^{2 n}\right\}$. Hence, applying Theorem 3.2 to $[\omega]=u^{\ell}+v^{\ell}$ the same way we did it in the previous example, we conclude that $\kappa\left(u^{\ell}+v^{\ell}\right)=(-1)^{\ell} c^{\ell}$ for $\ell<k$ and $\kappa([\omega])=0$ form any other $[\omega]$.

4.8. Orbifolds. Finally we will explain how the above results could be transformed to apply them to the orbifold case. Orbifolds have been studied extensively by many different authors, for example, a treatment of orbifold theory could be found in Satake [17], Kawasaki [12], Lerman-Tolman [15]. We will adopt the definitions and notations of Meinrenken [16] and will not go into any details of orbifold theory.

We just want to remind the reader that it is possible to define suborbifolds, smooth group actions on orbifolds and orbibundles. The definitions copy the same definitions in manifolds setting, taking into account local actions of finite groups. If a compact group $G$ acts locally freely on an orbifold $M$, then $M / G$ is again an orbifold. It is also possible to define vector fields, smooth forms, de Rham cohomology, characteristic classes and prove the analogue of the Stokes theorem for orbifolds. Moreover, it is possible to transform all these notions into the equivariant setting.

Given an orbifold $M$ with a smooth $G$ action, we again say that $M$ is equivariantly formal if its equivariant cohomology ring is torsion-free.

For every $x$ in $M$ there exists a unique up to isomorphism stabilizer group $H_{x}$ which fixes $x$ in every local chart and if $M$ is connected we define $d_{M}$ to be the minimal order of all such stabilizers. Actually $M$ could be stratified into smooth manifolds, such that points inside of every stratum have isomorphic stabilizers. If $M$ is connected there exists a unique open dense stratum with the order of its stabilizer being $d_{M}$.

This integer $d_{M}$ will be responsible for all the differences between orbifold and manifold cases of our theorems. In particular, taking this constant into account, the equation (1) changes to

$$
\frac{1}{d_{E}} \int_{E} a f_{*}(b)=\frac{1}{d_{Y}} \int_{Y} f^{*}(a) b .
$$

which leads to the following new formulation of Theorem 3.2 for a connected equivariantly formal orbifold $M$ and its suborbifold $W$ with a locally free circle action. 
Theorem 4.9. For any equivariantly closed forms $\alpha \in \Omega_{G / S^{1}}^{*}(X)$ and $\omega \in$ $\Omega_{G}^{*}(M)$ such that $\operatorname{deg} \alpha+\operatorname{deg} \omega \leq \operatorname{dim} W$

$$
\frac{(-1)^{\operatorname{deg} \omega+1}}{d_{X}} \int_{X} \frac{\kappa([\omega])[\alpha]}{e(W)}=\sum_{F_{j}} \frac{1}{d_{F_{j}}} \int_{F_{j}} \frac{\left[i_{j}^{*}(\omega \mu)\right]}{e\left(N F_{j}\right)},
$$

where $e(W)$ is the equivariant Euler class of the circle orbibundle $W \rightarrow X$ and $e\left(N F_{j}\right)$ are the equivariant Euler classes of the normal orbibundles $N F_{j}$ of $F_{j}$.

The proof of this theorem is the same as for Theorem 3.2 with only minor changes. Similarly, we can reformulate and prove Theorem 4.3 in the orbifold setting without any difficulties.

\section{Acknowledgments}

The author is grateful to his advisor Victor Guillemin for his constant support and guidance. The author thanks Maxim Braverman for his helpful and insightful remarks about an earlier version of this paper.

\section{References}

[1] M. F. Atiyah and R. Bott, The Yang-Mills equations over Riemann surfaces, Philos. Trans. Roy. Soc. London Ser. A 308 (1982), 523-615.

[2] _ The moment map and equivariant cohomology, Topology 23 (1984), 1-28.

[3] M. F. Atiyah and I. G. MacDonald, Introduction to commutative algebra, AddisonWesley Publishing Co., Reading, Mass.-London-Don Mills, Ont., 1969.

[4] N. Berline, E. Getzler, and M. Vergne, Heat kernels and Dirac operators, SpringerVerlag, Berlin, 1992.

[5] N. Berline and M. Vergne, Classes caractéristiques équivariantes. Formule de localisation en cohomologie équivariante, C. R. Acad. Sci. Paris Sr. I Math. 295 (1982), $539-541$.

[6] V. Ginzburg, V. Guillemin, and Y. Karshon, Assignments and abstract moment maps, J. Differential Geom. 52 (1999), 259-301.

[7] M. Goresky, R. Kottwitz, and R. MacPherson, Equivariant cohomology, Koszul duality, and the localization theorem, Invent. Math. 131 (1998), 25-83.

[8] V. Guillemin and J. Kalkman, The Jeffrey-Kirwan localization theorem and residue operations in equivariant cohomology, J. Reine Angew. Math. 470 (1996), 123-142.

[9] V. Guillemin and S. Sternberg, Supersymmetry and equivariant De Rham theory, Springer Verlag, Berlin, 1999.

[10] L. Jeffrey and F. Kirwan. Localization for nonabelian group actions, Topology 34 (1995), 291-327.

[11] J. Kalkman, Cohomology rings of symplectic quotients, J. Reine Angew. Math. 458 (1995), 37-52.

[12] T. Kawasaki, The index of elliptic operators over $V$-manifolds, Nagoya Math. J. 84 (1981), 135-157.

[13] F. Kirwan, Cohomology of quotients in symplectic and algebraic geometry, Mathematical Notes 31, Princeton University Press, Princeton, N.J., 1984.

[14] E. Lerman, Symplectic cuts, Math. Res. Lett. 2 (1995), 247-258.

[15] E. Lerman and S. Tolman, Hamiltonian torus actions on symplectic orbifolds and toric varieties, Trans. Amer. Math. Soc. 349 (1997), 4201-4230.

[16] E. Meinrenken, Symplectic surgery and the Spin ${ }^{c}$-Dirac operator, Adv. Math. 134 (1998), 240-277. 
[17] I. Satake, The Gauss-Bonnet theorem for V-manifolds, J. Math. Soc. Japan 9 (1957), $464-492$.

[18] E. Witten, Two-dimensional gauge theories revisited, J. Geom. Phys. 9 (1992), 303368.

Department of Mathematics, Northeastern University, Boston, MA 02115.

E-mail address: misha@research.neu.edu 